\title{
Mechanistic study of the formation of multiblock $\pi$-conjugated metallopolymer
}

\author{
Camila R. Peverari ${ }^{a}$, Diego N. David-Parra ${ }^{b}$, Madalina M. Barsan ${ }^{\text {, }}$, Marcos F.S. Teixeira ${ }^{a, *}$ \\ a Department of Chemistry and Biochemistry, Faculty of Science and Technology, São Paulo State University (UNESP), Rua Roberto Simonsen 305, CEP 19060-900 Presidente \\ Prudente, SP, Brazil \\ b São Carlos Institute of Chemistry, University of São Paulo (USP), São Carlos, SP, Brazil \\ ${ }^{\mathrm{c}}$ Department of Chemistry, Faculty of Science and Technology, University of Coimbra, 3004-535 Coimbra, Portugal
}

\section{A R T I C L E I N F O}

\section{Article history:}

Received 11 April 2016

Accepted 13 June 2016

Available online 17 June 2016

\section{Keywords:}

Electropolymerization

Metallopolymer

$\pi$-Conjugated polymer

Molecular columns

Molecular conductivity

\begin{abstract}
A B S T R A C T
Electropolymerization of a $\pi$-conjugated polymer from $\left[N, N^{\prime}\right.$-bis(salicylidene)-o-phenylenediamine $]$ nickel(II) complex ([Ni(salophen)]) on the surface of indium-tin-oxide (ITO) was studied. Two structures of the polymer have been proposed based on the theorem of molecular columns. The highlighted structures are the molecular column of the central aromatic rings (MCCAR) and molecular column of the side aromatic rings (MCSAR). The molecular structures were obtained by control of the potential scan rate in the electropolymerization step. The MCCAR structures are stable and structurally compact, whereas the MCSAR structures are less compact. Electrochemical measurements, atomic force microscopy (AFM), UVVis spectroscopy, FTIR Raman spectroscopy and optical microscopy support the formation of the multiblock $\pi$-conjugated polymer.
\end{abstract}

(c) 2016 Elsevier Ltd. All rights reserved.

\section{Introduction}

Schiff bases are widely employed in the development of new materials for nanotechnology. These molecules form typically stable ligands due the $p i$ bonds of imine units attached to aryl groups [1].

Goldsby and collaborators were among the first to report the formation of the poly(Schiff base) on a platinum surface [2,3]. Goldsby [4] and Audebert [5-7] described reasonably the electropolymerization mechanism for the transition Schiff base complex as a radical-radical coupling reaction. In this model, the polymers are considered as polaronic conductors without the participation of the central metals in the charge-transfer process and without variation of the oxidation state of the metal. However, Vilas-Boas et al. $[8,9]$ described a theorem of the electropolymerization process occurring by interactions of the electron-rich $\pi$ system with the metal center of the adjacent complex, functioning as bridges between the molecules. This model fits the data from previously reported research [10-12], due to the "donor-acceptor" interactions between the $\pi$-conjugated unit and the transition metal ion in the adjacent monomer. The conductivity of these polymers is related to the electronic transfer (ET) from a metallic cation to the aromatic $\pi$-conjugated systems (inner-sphere ET through

\footnotetext{
* Corresponding author. Fax: +55 1832215682 .

E-mail address: funcao@fct.unesp.br (M.F.S. Teixeira).
}

sequential hopping) $[13,14]$. In this way, the electropolymerization of the [ $N, N^{\prime}$-bis(salicylidene)-o-phenylenediamine]nickel(II) complex results in positively charged structures that form molecular columns, the positive charges being stabilized by negative ions inserted from the supporting electrolyte (counter ions) in the cavities of the polymers, which results in the formation of highly ordered and regular structures [8]. However, since each metal complex is formed by three aromatic moieties located on each side of the molecule center and one central (Fig. 1), mainly two different metallopolymer structures may occur on the conductor surface, both classified as molecular columns. This work was aimed to investigate the electrochemical processes of the conjugated metallopolymer structures based on [ $N, N^{\prime}$-bis(salicylidene)-ophenylenediamine]nickel(II) on an ITO surface. The studies comprise cyclic voltammetry (CV), electronic conductivity, atomic force microscopy (AFM), UV-Vis, Raman spectroscopy and optical microscopy.

\section{Experimental}

\subsection{Synthesis of salophen ligand and [Ni(salophen)] complex}

The ligand $N, N^{\prime}$-bis(salicylidene)-o-phenylenediamine (salophen) $[15,16]$ was obtained by reaction of salicylaldehyde (18 mmol) and 1,2-phenylenediamine $(9 \mathrm{mmol})$ in absolute 


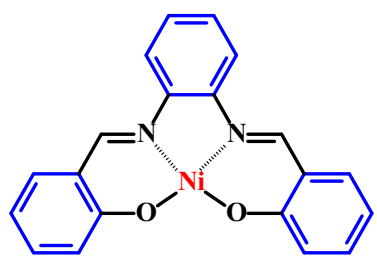

Fig. 1. Molecular structure of [ $N, N^{\prime}$-bis(salicylidene)-o-phenylenediamine]nickel(II) in highlighted aromatic moieties.

ethanol and kept under reflux for $15 \mathrm{~h}$ at $40^{\circ} \mathrm{C}$ to produce a yellow precipitate. It was filtered, washed and recrystallized with absolute ethanol and finally dried in desiccator. The metallic complex [Ni (salophen)] was prepared by addition of stoichiometric and equimolar amounts of the Schiff base ligand $(2.86 \mathrm{mmol})$ and nickel acetate in absolute ethanol. The solution was kept under reflux for $3 \mathrm{~h}$ at $50{ }^{\circ} \mathrm{C}$ with constant stirring to yield a red precipitate. The precipitate was filtered in a Gooch crucible, washed with absolute ethanol and kept in a desiccator. The yield of the complex was about 85\%. FTIR $\left(v \mathrm{~cm}^{-1}\right)$ in $\mathrm{KBr}: 3339-\mathrm{OH}_{\mathrm{ph}} ; 1620 \mathrm{C}=\mathrm{N} ; 1600$ $\mathrm{C}=\mathrm{C}, 1362>\mathrm{C}-\mathrm{O}_{\mathrm{ph}}, 1023$ breathing ring, $765 \mathrm{Ni}-\mathrm{O}$. UV-vis $(\mathrm{nm})$ in $\mathrm{CH}_{3} \mathrm{CN}: 310\left(\pi \rightarrow \pi^{*}\right), 330\left(\pi \rightarrow \pi^{*}\right), 375\left(\mathrm{n} \rightarrow \pi^{*}\right), 473$ (MLCT).

\subsection{Metallopolymer synthesis}

Synthesis of poly[Ni(salophen)] on a conductive substrate was performed by cyclic voltammetry. Measurements were carried out in a conventional electrochemical cell, with a saturated calomel electrode (SCE) as reference electrode, platinum electrode as counter electrode and indium tin oxide (ITO) coated glass (area $=1.0 \mathrm{~cm}^{2}$ ) as working electrode, connected to a potentiostat/ galvanostat $\mu$ Autolab type III. Films of different thickness were prepared by use of different potential cycles and scan rates. The ITO/glass conductors were cleaned by sonication for $30 \mathrm{~min}$., first in acetone then in isopropyl alcohol to remove any residual organic contamination.

The metallopolymer was electrodeposited by continuous oxidative potential scans on conductor substrate (working electrode) between 0.1 and $1.5 \mathrm{~V}$ versus SCE, immersed in a benzonitrile solution containing $5.0 \mathrm{mmol} \mathrm{L}^{-1}$ of [Ni(salophen)] monomer and $0.1 \mathrm{~mol} \mathrm{~L}^{-1}$ tetrabutylammonium perchlorate (supporting electrolyte) under a nitrogen atmosphere. (CAUTION: Due to the high toxicity of benzonitrile, measurements were performed in a fume hood)

\subsection{Characterization of the metallopolymer}

Electrochemical characterization of the metallopolymer on the ITO was realized by cyclic voltammetry in aqueous solution of $\mathrm{KCl} 0.5 \mathrm{~mol} \mathrm{~L}^{-1}$. The electrochemical study was carried out in aqueous solution to minimize the effect of ohmic resistance of the electrolytic medium. Conductivity measurements were performed by linear sweep voltammetry at $25 \mathrm{mV} \mathrm{s}^{-1}$. Current-potential profiles were generated by measuring the current as the voltage was incremented from 0.35 to $0.85 \mathrm{~V}$. Conductivity profiles were constructed by the difference between faradaic current density and capacitive current density, and converted to electronic conductivities by applying Ohm's law:

$\sigma=\frac{j\left(A c m^{-2}\right) \times d(\mathrm{~cm})}{\Delta E(V)}$

where $\Delta E$ is applied potential, $j$ is current density and $d$ is layer thickness of the polymer.
UV-Vis spectroscopy measurements were carried out with a Perkin Elmer Lambda 25 spectrophotometer to determine the characteristic absorption band and to monitor the growth of the metallopolymer deposited onto the ITO substrate. Solid-state absorption spectra of all samples were obtained in a dry and neutral state (without potential application). Raman spectroscopy measurements were obtained with a Renishaw Raman spectrometer with laser at an excitation wavelength of $663 \mathrm{~nm}$ and a diffraction grating of 1800 lines per millimeter. The exposure time was set at $10 \mathrm{~s}$ with three accumulations.

Morphologic features of the metallopolymer were analyzed by atomic force and optical microscopy. Atomic force microscopy images of films were captured with a Nanosurf (Boston MA) EasyScan 2 AFM. The scan head imaged at an angle 45 degrees relative to the axis of stretching. Collected images were analyzed with Nanosurf AFM software (v. 3.0.2.4) to determine roughness and thickness. Optical microscopy was accomplished with an optical microscope (Leica - DMLM series) coupled to a spectrograph, whose objective had a $50 \times$ increase in providing a spatial resolution of around $1.0 \mu \mathrm{m}^{2}$, Peltier CCD detector (cooled to $-70^{\circ} \mathrm{C}$ ) and motorized XYZ platform (stepper motor $-0.1 \mathrm{~mm}$ ), where the samples were positioned.

\section{Results and discussion}

\subsection{Electropolymerization and electrochemical properties of the metallopolymer}

In the electropolymerization step, different solvents were tested to find a suitable medium for the film fabrication, i.e., carbon tetrachloride, hexane, chloroform, dimethylformamide, acetone, dichloromethane, acetonitrile and benzonitrile. It was observed that solvents with high dipole moments, such as acetonitrile $(\mu=3.53 \mathrm{D})$ and benzonitrile $(\mu=4.01 \mathrm{D})$ [17] lead to better polymer formation, explained as follows: since the electropolymerization reaction occurs due to formation of a short half-life cation radical, solvents with high dielectric constant and low viscosity stabilize better the radical. Due to the increasing concentration of cation radical on the ITO conductor substrate and consequently increased polarity of the oligomeric molecules, benzonitrile was the best solvent for obtaining the metallopolymer. The influence of supporting electrolyte tetrabutylammonium perchlorate (TBAP) and tetrabutylammonium hexafluorophosphate $\left(\mathrm{TBAPF}_{6}\right)$ in the electropolymerization were also investigated. Timonov et al. [1820] have described the influence of the counter-anion size of the supporting electrolyte during electropolymerization on the voltammetric characteristics. The distribution of the molecular column over the conductor surface may be controlled by use of charge-compensating ions from the supporting electrolyte of different ionic radii. During the formation of the metallopolymer, the molecular column acquires a positive charge during scanning oxidation, which is compensated by anions from the supporting electrolyte. Between the two tested supporting electrolytes, tetrabutylammonium perchlorate (TBAP) was more suitable for the metallopolymer synthesis. In the $\mathrm{TBAPF}_{6}$ supporting electrolyte, the formation of the polymeric film was not observed due to the small size of the hexafluorophosphate anion $(0.198 \mathrm{~nm})$ [21], hindering the formation of the molecular columns which are too close, causing greater repulsion during electropolymerization. The perchlorate anion $(0.241 \mathrm{~nm})$ [21] fits perfectly, providing an ideal spacing in between the molecular columns, and improves the conductivity of the polymer providing a more efficient electropolymerization.

Polymer film formation on the ITO surface was carried out by successive potential cycling at $200 \mathrm{mVs}^{-1}$ in benzonitrile medium. 
Fig. 2A shows that successive scans produce an increase of both anodic and cathodic peak current. For subsequent studies it was established that eight potential cycles are sufficient to obtain a metallopolymeric structure with good ionic conductivity. Fig. 2B shows one redox couple that occurs in the electropolymerization step and can be assigned to the $\mathrm{Ni}(\mathrm{II}) / \mathrm{Ni}(\mathrm{III})$ process [23,32-35]. The anodic wave centered at approximately $0.72 \mathrm{~V}$ versus SCE, associated with the $\mathrm{Ni}(\mathrm{II}) / \mathrm{Ni}(\mathrm{III})$ couple (Eqs. (1) and (2)) in organic solvent, is consistent with the behavior reported by Teixeira et al. $[11,12]$ for a model "molecular column" and where the metallopolymer containing nickel complexes are bound to all available coordination sites on the polymer backbone.

poly[Ni(II)salophen $]_{(\text {ITO })} \rightarrow$ poly[Ni(III)salophen $]_{(\text {ITO })}^{+}+\mathrm{e}^{-}$

poly[Ni(III)salophen $]_{(\text {ITO })}^{+}+\mathrm{e}^{-} \rightarrow$ poly[Ni(II)salophen $]_{(\text {ITO })}$

Polymeric film formation on a conductive surface occurs during oxidation of the central metal cation. Transition metals have the ability to share electron density with $\pi$-systems through $d$-orbitals. On the basis of this fact, the formation mechanism of the polymeric film on conductor surface can be ascribed to the interaction between $d$-orbitals of the metal centers with $\pi$-orbitals of aromatic rings of adjacent monomers, similar to the sandwich structure formation [22], which is best achieved when monomers of squareplanar spatial structure are used. It was reported in our previous studies $[10,11,23]$ and by others $[18,19,24]$, that the structure of a salen ligand containing metallopolymer is formed between the face of an electron-rich $\pi$ system and an adjacent central cation of another complex. The existence of three aromatic ring moieties in the salophen ligand structure (see Fig. 1) enables the formation of mainly two different molecular structures on the conductor surface through interactions between the metal cation and $\pi$ system that can be classified as molecular columns structure. This was evidenced by the cyclic voltammetric profile of the metallopolymer onto the ITO surface in aqueous solution that exhibited two wellseparated redox couples with midpoint potentials at $+0.31 \mathrm{~V}$ and $+0.59 \mathrm{~V}$ versus SCE that are assigned to the redox process of $\mathrm{Ni}$ (II)/Ni(III) (Supplementary material - MS1). The potential gap between the redox pairs arises from changes in the electron density of the polymer backbone, which suggests the existence of two different polymer structures with distinct electronic communication between the nickel centers. Malev and collaborators [24] studied the possible causes responsible for the fact that cyclic voltammograms of polymer nickel-Schiff base possess some shoulders or are split into two peaks, although the total electrode process has a one-electron character. The research conducted a quantitative analysis of electrochemical response and indicated a mechanism based on the mobility of charge (polaron/bipolaron conductance and disproportionation reaction of polarons). The coverage of the metallopolymer on the ITO surface was confirmed by the linear dependence of the anodic and cathodic peak currents with the potential scan rates $\left(10-200 \mathrm{mV} \mathrm{s}^{-1}\right)$. This showed that the redox processes were controlled by an adsorption mechanism on the conductor surface and considered as a Nernstian reaction. In adsorption processes, the concentrations of the electroactive species $(\Gamma)$ on the surface can be calculated by a method reported by Sharp et al. [25], based on which the surface coverages of the metallopolymer were about 0.7 and $0.2 \mathrm{nmol} \mathrm{cm}^{-2}$ for redox couples at 0.31 and $0.59 \mathrm{~V}$ versus SCE, respectively.

To understand the formation of the two types of molecular columns, the influence of the electropolymerization scan rate (5$200 \mathrm{mV} \mathrm{s}^{-1}$ ) on the concentration of electroactive species onto the surface was studied, and it was observed that an increase of the scan rate led to a decrease in electroactive species concentration on the ITO surface (Supplementary material - MS2). For a high scan rate, the polymer backbone is less stable, since there is not sufficient time to generate enough cation radicals on the conductor interface; consequently, the formation of metallopolymer structures is hindered. Lower scan rates were more efficient for polymer coating. Surprisingly, the cyclic voltammogram in an aqueous solution of the metallopolymer formed at $5 \mathrm{mV} \mathrm{s}^{-1}$ (Supplementary material - MS3), exhibited only a redox couple at $+0.45 \mathrm{~V}$ versus SCE, evidencing that at a low electropolymerization scan rate there is a predominance of one of the molecular columns configurations, which enables electronic communication between the nickel centers in the conjugated backbone. The surface coverage of the metallopolymer formed at $5 \mathrm{mV} \mathrm{s}^{-1}$ was $35.2 \mathrm{nmol} \mathrm{cm}{ }^{-2}$.

The study of electronic conductivity can provide significant information about the charge-transport mechanism of poly[ $\mathrm{Ni}$ (salophen)] produced by using the two electropolymerization scan rates studied (5 and $200 \mathrm{mV} \mathrm{s}^{-1}$ ). According to Abruña [26,27], the measurements of charge transport at chemically modified electrodes are related to physical movement of species, electron selfexchange, morphology of the deposited layer, and counterion transport. The conductivity profiles of poly[Ni(salophen)] shown in Fig. 3 are also indicative of redox behavior. The currents have been converted to electronic conductivities by applying Ohm's law and adjusted for the dimensions of the metallopolymer film. The metallopolymer produced at $200 \mathrm{mV} \mathrm{s}^{-1}$ presented two peaks in the conductivity profile corresponding to the two redox pairs observed in the cyclic voltammetry (Fig. $3 \mathrm{~A}$ ). The difference in
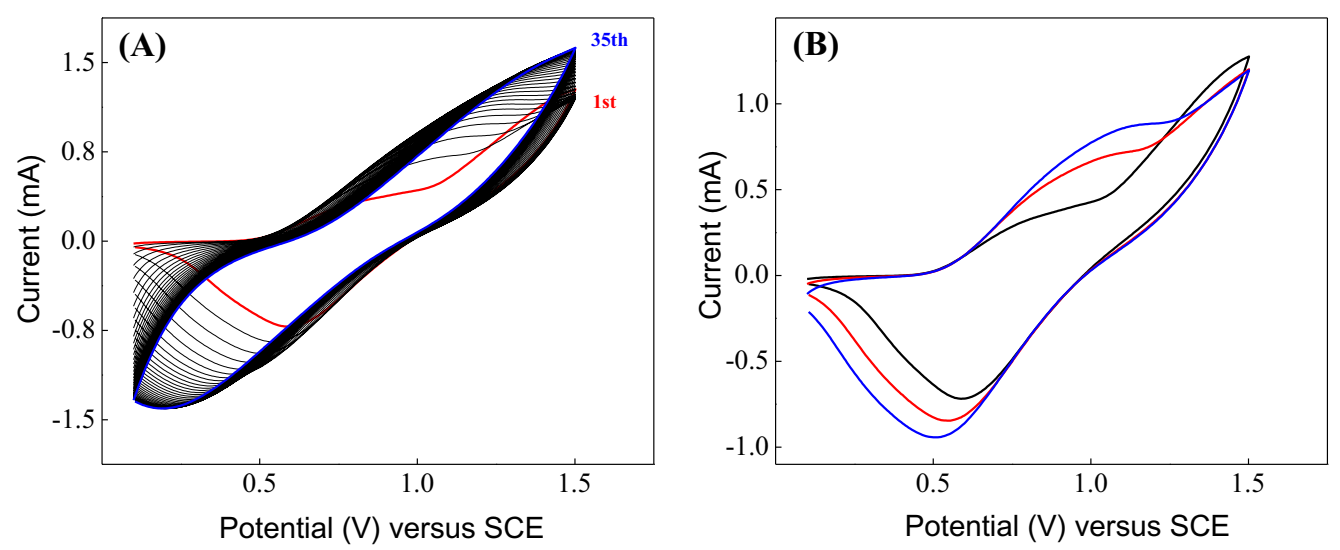

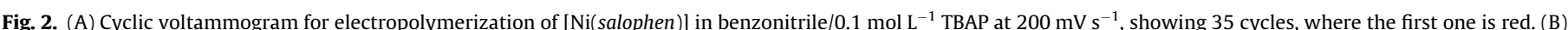

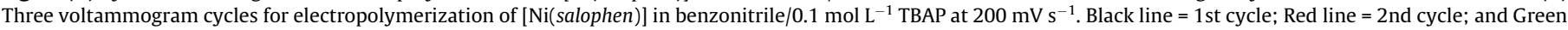
line $=3$ rd cycle. (Colour online.) 


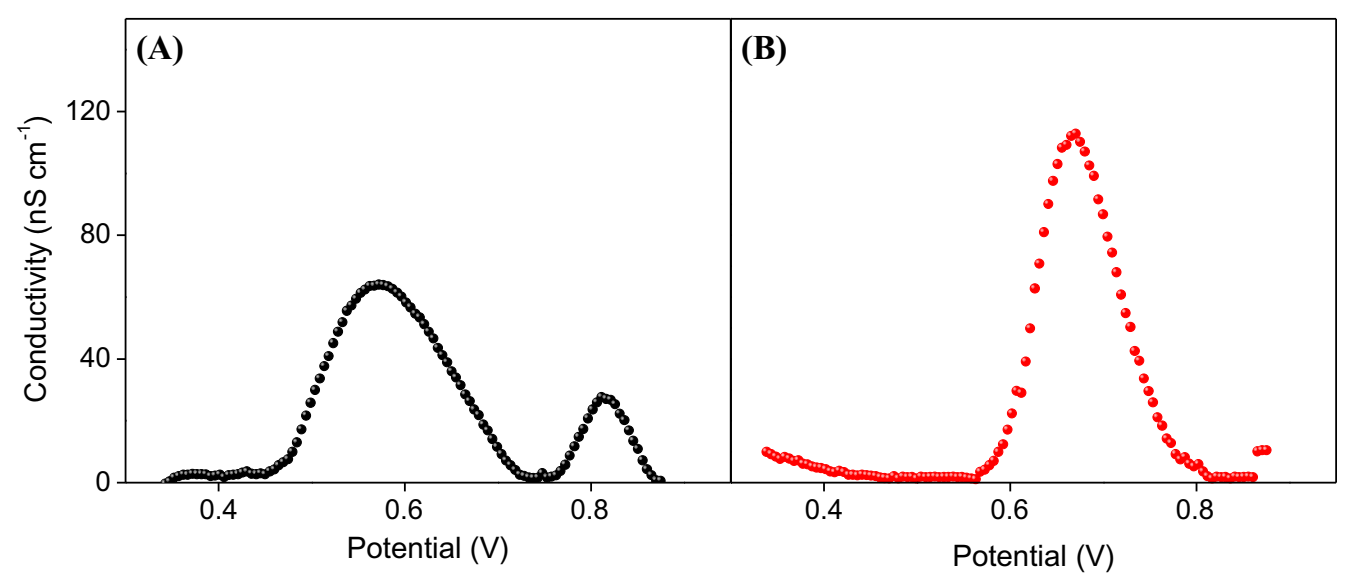

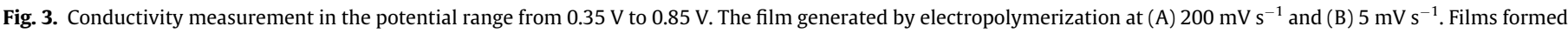
by 35 cycles. $T=298 \mathrm{~K}$.

magnitude of the two conductivity peaks is consistent with changes in coordination of the metallopolymer complexes and indicates a faster electron transport at $0.52 \mathrm{~V}$, indicating molecular structures with good electronic communication between cation centers. The smaller peak at $0.81 \mathrm{~V}$ shows that electronic conductivity via the redox sites on the polymer backbone is not the preferential electron-transfer pathway. These observations can be explained by the formation of the two different molecular column structures by interactions between the d-orbitals of nickel centers with $\pi$-orbitals of the aromatic rings of salophen adjacent, i.e., between the $d$-orbitals of the nickel with the $\pi$-orbital of the central aromatic rings of the adjacent salophen, forming molecular columns of central aromatic rings MCCAR (Scheme 1a) and d-orbitals of the nickel with $\pi$-orbital of phenolic rings of the salophen adjacent, yielding molecular columns of side aromatic rings MCSAR (Scheme 1b). Probably, the MCCAR structure are more compact

(a)

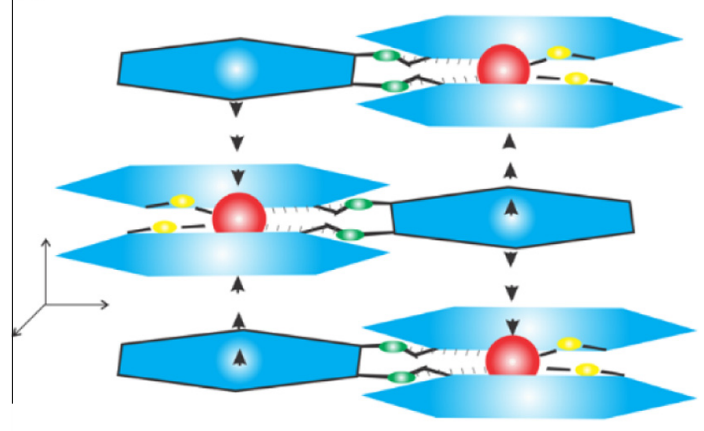

(b)

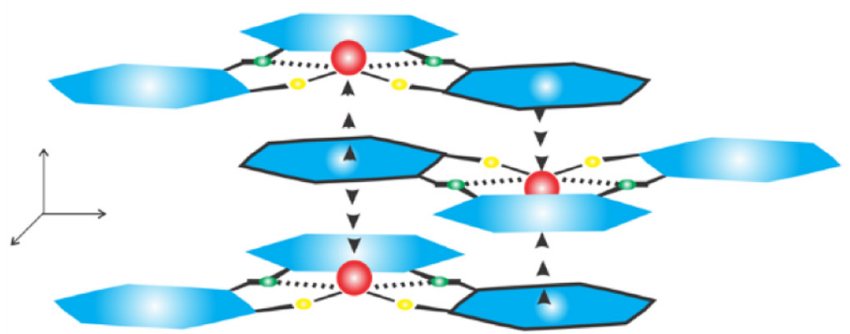

Scheme 1. Schematic representation of the spatial structures of (a) MCCAR (molecular columns of central aromatic rings) and (b) MCSAR (molecular columns of side aromatic rings). Red = nickel; Yellow = oxygen; Green = nitrogen; Cyan = aromatic ring. (Colour online.) than the MCSAR ones, since the $\pi$ electronic system is substantially compartmentalized in the molecular column, enabling better electronic communication between redox centers.

On the other hand, the conductivity profile of the metallopolymer formed at $5 \mathrm{mV} \mathrm{s}^{-1}$ (Fig. 3B) presented only one conductivity peak $\left(\sigma_{\max }=1.2 \times 10^{-7} \mathrm{~S} \mathrm{~cm}^{-2}\right)$ at $0.65 \mathrm{~V}$, indicating a higher relative conductivity in comparison with the film obtained at $200 \mathrm{mV} \mathrm{s}^{-1}$. By conductivity investigations, we conclude that an electropolymerization scan rate of $5 \mathrm{mV} \mathrm{s}^{-1}$ produces a polymer film with a single spatial structure that enables rapid electron transfer between localized redox states in the polymer backbone. Probably, the predominant structure for this type of polymer is MCCAR as we have previously observed in the study of voltammetry.

\subsection{UV-Vis absorption spectroscopy}

The aim of the UV-Vis spectroscopy study was to investigate further the effect of scan rate on the structural differences of the synthesized metallopolymer. The intense color obtained on the conductor surface with successive cycling (from the 2nd to the 8th cycle) was the first evidence of the film formation (Supplementary material - SM4). Substrates coated with films at $200 \mathrm{mV} \mathrm{s}^{-1}$ remained optically transparent, whereas the ones at $5 \mathrm{mV} \mathrm{s}^{-1}$ turned dark brown and more opaque. The absorption spectra measurements at ITO with two different coatings were carried out at room temperature for visible wavelengths ranging from 330 to $760 \mathrm{~nm}$ in the neutral state of the metallopolymer and are shown in Fig. 4.

Solid-state absorption spectra of all the samples obtained at $200 \mathrm{mV} \mathrm{s}^{-1}$ gave essentially the same profile (Fig. 4 A) with an absorption maximum at $375 \mathrm{~nm}$ corresponding to the $\pi-\pi^{*}$ transition of the metallopolymer due to aromatic rings, and the band gap was calculated to be $2.7 \mathrm{eV}$ from this transition. The most significant absorptions are a metal-to-ligand charge-transfer band MLCT at $491 \mathrm{~nm}$. Absorbance values do not increase linearly with the number of electrodeposition cycles as expected; a more detailed analysis of the polymeric film thickness investigated by atomic force microscopy is presented in Section 3.4. Moreover, no displacement of peak bands with an increase in the number of ligand and metal centers during the electropolymerization was observed [28]. This indicates the existence of film structures with low interaction between polymer layers with a minimized overlapping of the orbitals (Supplementary material - SM5). Hence, the electronic transfer is not significant through the polymer film and 


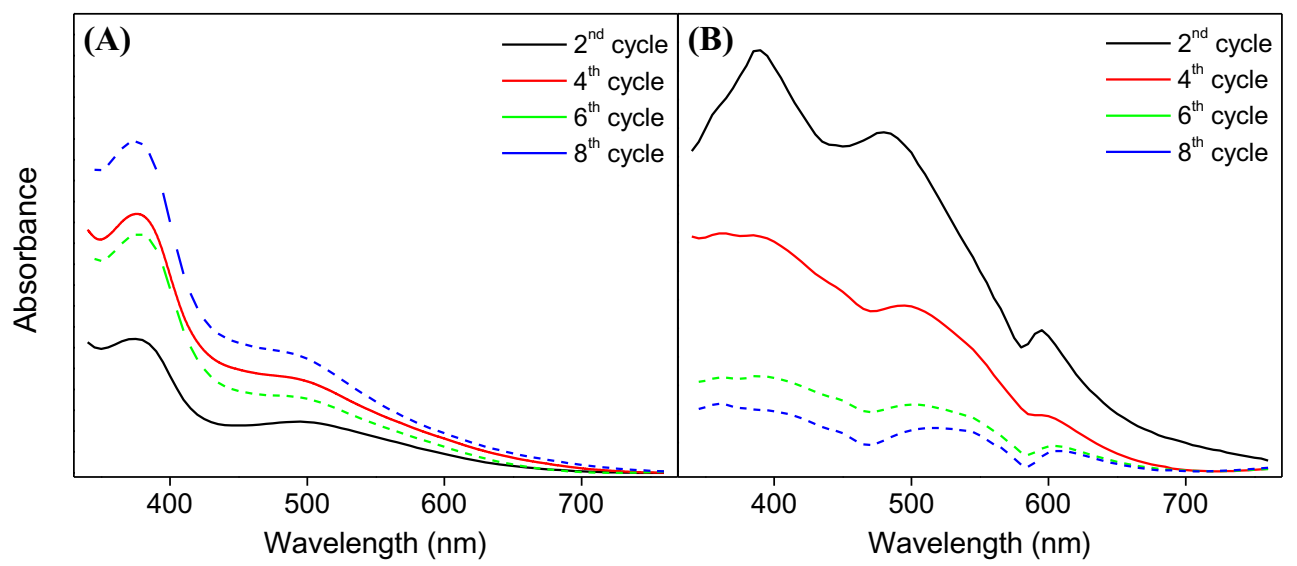

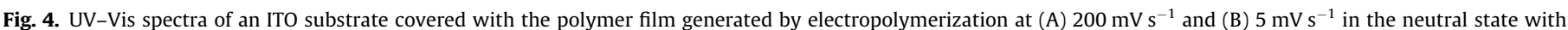
different electrodeposition cycles.

consequently the shift of wavelength is negligible for films with different thicknesses.

The substrate covered with the metallopolymer obtained at $5 \mathrm{mV} \mathrm{s}^{-1}$ presented a different spectrum than that obtained at $200 \mathrm{mV} \mathrm{s}^{-1}$, with two bands at 388 and $480 \mathrm{~nm}$, plus a new absorption band at $593 \mathrm{~nm}$ (Fig 4B). From the onset of the absorption maximum ( $\lambda_{\text {onset }}$ ), the optical band gaps were estimated to be of $2.15,1.98$ and $1.86 \mathrm{eV}$, respectively. The band gaps increased for lower energies, this behavior reflects the electronic changes in the HOMO and LUMO levels in backbone polymer.

The MLCT band showed simultaneously a bathochromic shift and broadening, with the increase in polymerization cycle number, associated with a change in the polarity of the environment of this MLCT charge-transfer band [29-32], and to the extension of the $\pi$ conjugation bonds. The interaction between d-orbitals of nickel centers with $\pi$-orbitals of the aromatic ring of salophen is initially compartmentalized in the solid state $(388 \mathrm{~nm})$, which then, with increasing polymerization cycles, gradually merges with the other at $480 \mathrm{~nm}$, suggesting that $\pi$ electrons are delocalized along the polymer backbone. This delocalization implies stronger interactions between the central aromatic rings of the salophen adjacent to the coordination site of nickel. Broadening of this MLCT is associated with the electrogeneration of different oligomers with similar electronic properties, whereas the new band (between 590 and $610 \mathrm{~nm}$ ) is considered to originate from d-d transition [33,34], indicating the existence of axial coordination with the central metal cation $\left(\mathrm{d}^{8}\right.$ square-planar $\left.\mathrm{Ni}^{\mathrm{II}}\right)$ and consequently the formation of three-dimensional networks.

\subsection{FTIR resonance Raman spectra}

Raman spectroscopy has proved to be important in the analysis of metal sandwich species, in the elucidation of metal cation- $\pi$ system interactions $[35,36]$. The connection between the electronic structure deduced from UV-Vis spectra and the geometrical structure supported by the vibrational data might be established from of the Raman spectra. Fig. 5 presents Raman spectra obtained on the ITO with films obtained at 200 and $5 \mathrm{mV} \mathrm{s}^{-1}$ polymerization scan rates (Fig. 5B and C, respectively); for comparison the spectrum of the non-polymerized material is presented in Fig. 5A.

Analyzing the rotational and vibrational structures in the spectra, one can observe that the common bands associated with the stretching of $\mathrm{C}-\mathrm{O}$ and $\mathrm{C}=\mathrm{C}$ bonds in the $1600-1100 \mathrm{~cm}^{-1}$ region are very little affected. The major difference is the appearance of peaks in the $800-300 \mathrm{~cm}^{-1}$ region that can be attributed to the symmetrical metal-ring stretching in the polymer. The values are compatible with the ones obtained for metal sandwich species [37-40], providing evidence for the formation of the nickel- $\pi$ system in the polymer. The fact that the spectrum of the metallopolymer obtained at $5 \mathrm{mV} \mathrm{s}^{-1}$ has sharper peaks in this region indicates the predominance of a nickel- $\pi$ system in the polymer.

Another important aspect observed in the spectra of the metallopolymer is the strong suppression of the fluorescence background in comparison with the spectrum of the non-polymerized material. It is known that Schiff base complexes are fluorogenic materials and that fluorescence emission occurs with laser activation. This suppression was more evident for polymer obtained at $5 \mathrm{mV} \mathrm{s}^{-1}$ due to the $\mathrm{d}-\mathrm{d}$ transition of the metal as observed in the UV-Vis absorption spectrum (Section 3.1). The emitted energy, due to resonance between the emission bands of the complex induced by the laser, is reabsorbed by nickel to promote the electronic transition in the $\mathrm{d}-\mathrm{d}$ absorption band region, and consequently the decrease in fluorescence background occurs.

\subsection{Surface morphologic characterization}

Nanoscale morphology of the ITO covered with the metallopolymer was investigated by means of atomic force microscopy (AFM); the images are shown in Fig. 6, with the roughness and thickness values in Table 1 . Images and thickness values were obtained only for the metallopolymer obtained at $200 \mathrm{mV} \mathrm{s}^{-1}$, since the polymer film obtained at $5 \mathrm{mV} \mathrm{s}^{-1}$ showed roughness superior to $1 \mu \mathrm{m}$, preventing the AFM measurement. Fig. $6 \mathrm{~A}$ shows that the surface of the film is not smooth and contains cluster structures with diameters between 150 and $5000 \mathrm{~nm}$. These structures definitely suggest an amorphous morphology film, in agreement with results obtained in the conductivity and UV-Vis studies (Sections 3.1 and 3.2).

This thickness and roughness change with the increase in the electropolymerization cycle was investigated. The flatter areas with smaller roughness were obtained for films after 2 and 6 electrodeposition cycles; the film formed with eight cycles had a more granular, thicker and rougher morphology compared with films obtained during fewer polymerization cycles.

It is expected that the thickness of the polymeric film would increase linearly with number of electropolymerization cycles. However, there is evidence in the UV-Vis spectra of metallopolymer formation discontinuity on the conductor surface. A plausible explanation for such up-and-down changes of the thickness of polymer can be related to the instability of the stratification of molecular columns and consequently a "breakdown" structural. 


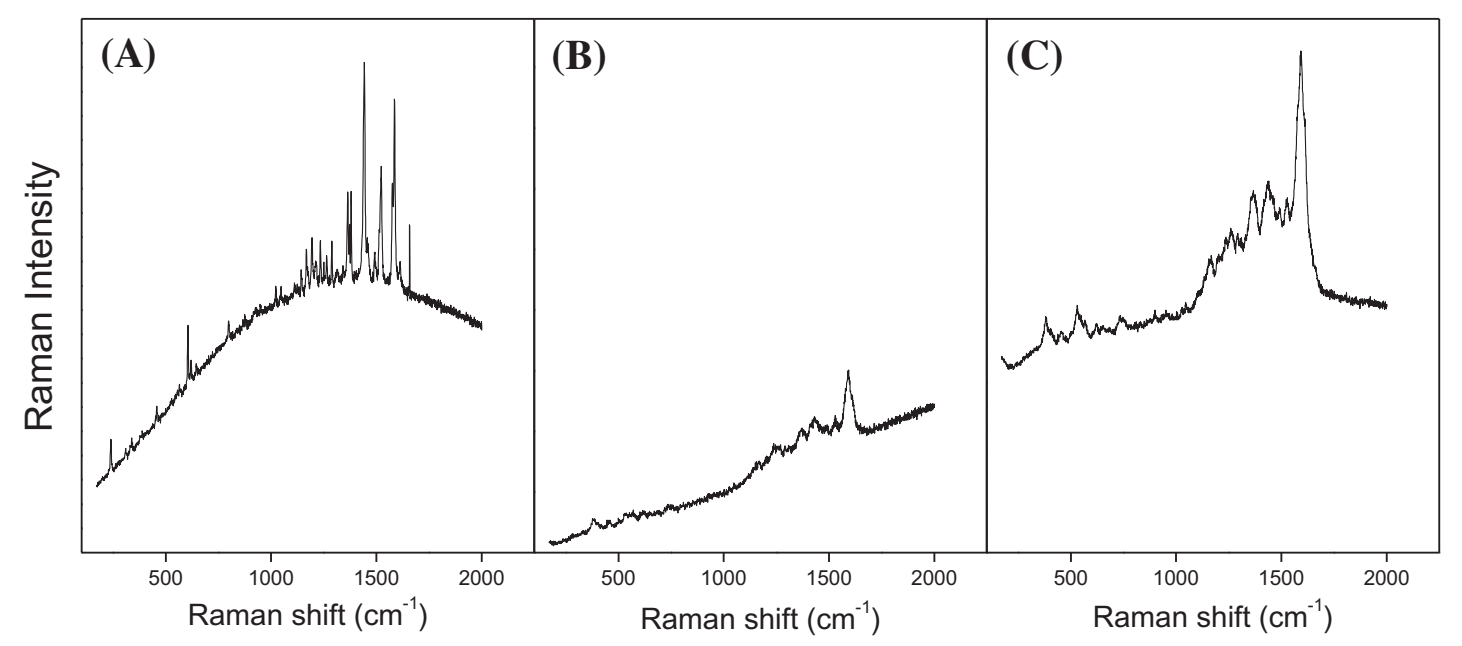

Fig. 5. Resonance Raman spectra of (A) non-polymerized material, (B) metallopolymer formed at $200 \mathrm{mV} \mathrm{s}^{-1} \mathrm{and}_{(\mathrm{C})} 5 \mathrm{mV} \mathrm{s} \mathrm{s}^{-1} \cdot \lambda_{\mathrm{ex}}=663 \mathrm{~nm}$.
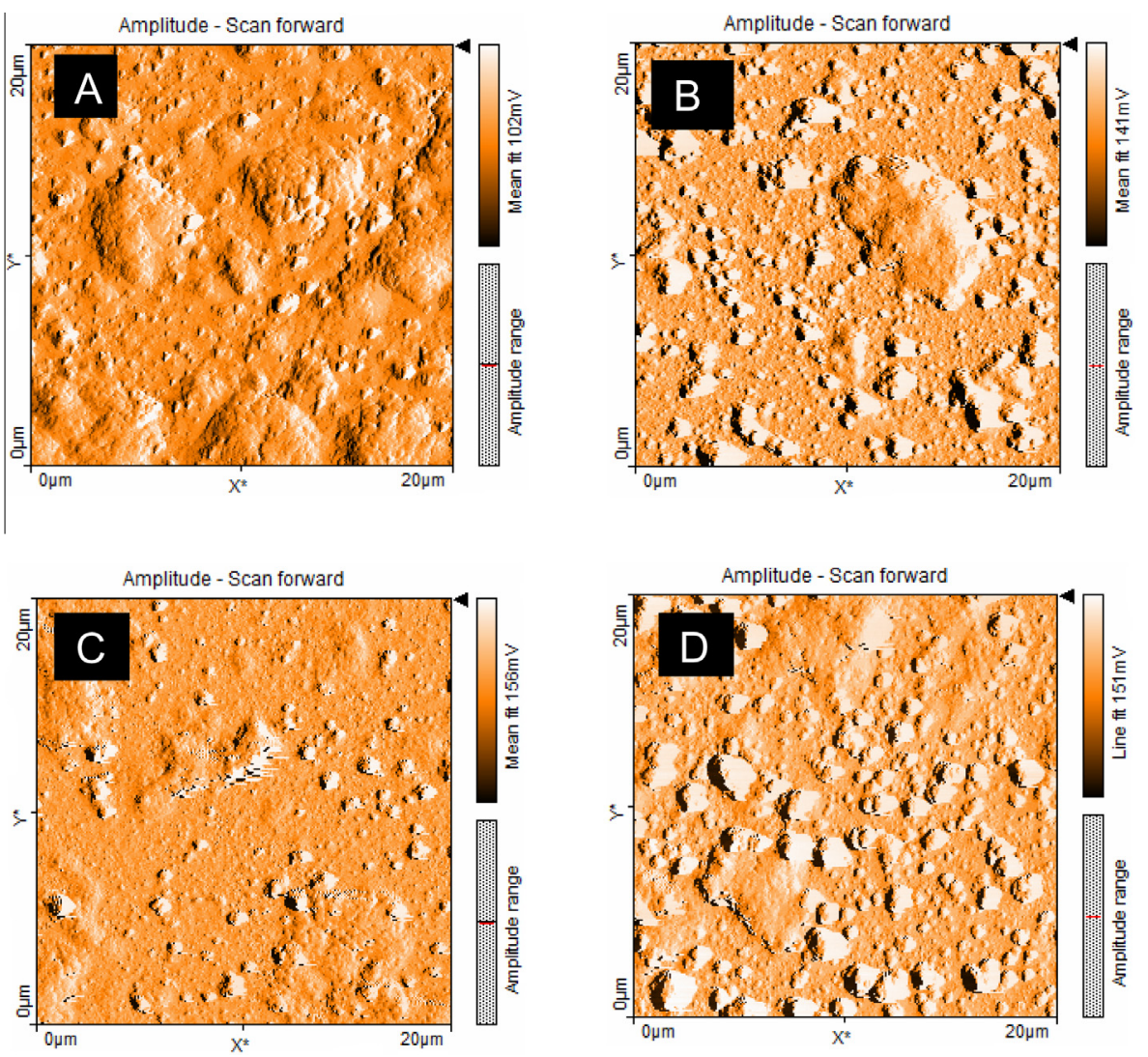

Fig. 6. AFM images of metallopolymer growth analyzed in (A) 2rd (B) 4th (C) 6th and (D) 8th electrodeposition cycle on an ITO surface.

Table 1

Values of thickness and roughness for the polymeric films formed at $200 \mathrm{mV} \mathrm{s}^{-1}$ in different electrodeposition cycles.

\begin{tabular}{lllll}
\hline Cycles & 2nd & 4th & 6 th & 8th \\
\hline Thickness $(\mathrm{nm})$ & 106 & 130 & 79.45 & 125.8 \\
Roughness $(\mathrm{nm})$ & 71 & 91 & 68 & 117 \\
\hline
\end{tabular}

Optical microscopy images shown in Fig. 7 reveal different textures of the two polymers on the surface of ITO obtained at $200 \mathrm{mV} \mathrm{s}^{-1}$ (Fig. 7A) and at $5 \mathrm{mV} \mathrm{s}^{-1}$ (Fig. 7B). The first image shows aggregates ranging from 1 to $2 \mu \mathrm{m}$ in width, with a very irregular and nonhomogeneous polymer surface, whereas the second image was more uniform and homogeneous with clear compact globular structures of the polymer.

\section{Conclusions}

In this work, we have demonstrated the formation of two structures based on the theorem of molecular columns. Highlighted structures consist of molecular columns of central aromatic rings (MCCAR) and molecular columns of the side aromatic rings (MCSAR). Molecular structures were obtained by control of the potential scan rate in the electropolymerization step. Electrochem- 

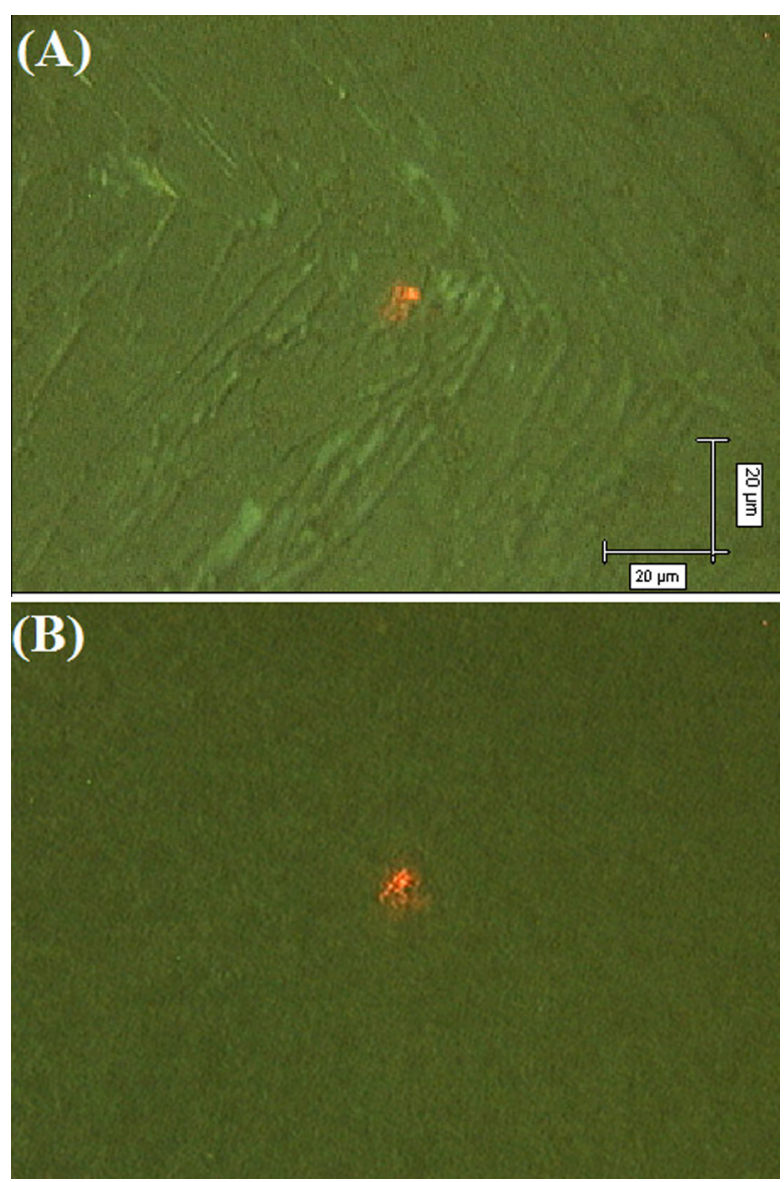

Fig. 7. Optical microscope images of the polymer surface deposited on ITO substrate obtained at (A) $200 \mathrm{mV} \mathrm{s}^{-1}$ and (B) $5 \mathrm{mV} \mathrm{s}^{-1}$ for 8 cycles.

ical measurements, atomic force microscopy (AFM), UV-Vis spectroscopy, FTIR Raman spectroscopy techniques and optical microscope images support the formation of the multiblock $\pi$ conjugated polymer. When structured polymer surfaces are required, the metallopolymer formed at $5 \mathrm{mV} \mathrm{s}^{-1}$ is a good candidate due to its compact structure and enhanced electrical conductivity. Taken together, the metallopolymer films represent a dynamic and intriguing approach in their formation, and a better understanding of the fundamental parameters of these assemblies will aid in their future applications.

\section{Acknowledgments}

The authors acknowledge FAPESP (2005/01296-4) and CNPq (137444/2010-9 and 234256/2014-1) for financial support. SJT $\therefore$

\section{Appendix A. Supplementary data}

Supplementary data associated with this article can be found, in the online version, at http://dx.doi.org/10.1016/j.poly.2016.06.021.

\section{References}

[1] O. Fatibello, E.R. Dockal, L.H. Marcolino, Anal. Lett. 40 (2007) 1825.

[2] D. Ramprasad, W.K. Lin, K.A. Goldsby, D.H. Busch, J. Am. Chem. Soc. 110 (1988) 1480.

[3] K.A. Goldsby, J.K. Blaho, L.A. Hoferkamp, Polyhedron 8 (1989) 113.

[4] K.A. Goldsby, J. Coord. Chem. 19 (1988) 83.

[5] P. Audebert, P. Capdevielle, M. Maumy, New J. Chem. 15 (1991) 235.

[6] P. Audebert, P. Capdevielle, M. Maumy, Synth. Met. 43 (1991) 3049.

[7] P. Audebert, P. Capdevielle, M. Maumy, New J. Chem. 16 (1992) 697.

[8] M. VilasBoas, C. Freire, B. deCastro, P.A. Christensen, A.R. Hillman, Inorg. Chem. 36 (1997) 4919.

[9] M. Vilas-Boas, C. Freire, B. de Castro, P.A. Christensen, A.R. Hillman, Chem. - A Eur. J. 7 (2001) 139.

[10] T.R.L. Dadamos, M.F.S. Teixeira, Electrochim. Acta 54 (2009) 4552

[11] C.S. Martin, T.R.L. Dadamos, M.F.S. Teixeira, Sens. Actuators B 175 (2012) 111.

[12] C.S. Martin, W.B.S. Machini, M.F.S. Teixeira, RSC Adv. 5 (2015) 39908.

[13] W.J. Liu, W.J. Huang, C.H. Chen, M. Pink, D. Lee, Chem. Mater. 24 (2012) 3650.

[14] B.J. Holliday, T.M. Swager, Chem. Commun. (2005) 23.

[15] V.Z. Mota, G.S.G. de Carvalho, P.P. Corbi, F.R.G. Bergamini, A.L.B. Formiga, R. Diniz, M.C.R. Freitas, A.D. da Silva, A. Cuin, Spectrochim. Acta Part A 99 (2012) 110.

[16] L. Carbonaro, A. Giacomelli, M. Isola, L. Senatore, J. Chem. Soc., Dalton Trans. (1992) 41.

[17] K.o. Izutsu, Electrochemistry in Nonaqueous Solutions, 2nd., rev. and enlarged ed., Wiley-VCH, Weinheim, 2009.

[18] T.Y. Rodyagina, P.V. Gaman'kov, E.A. Dmitrieva, I.A. Chepurnaya, S.V. Vasil' eva, A.M. Timonov, Russ. J. Electrochem. 41 (2005) 1101.

[19] S.V. Vasil'eva, I.A. Chepurnaya, S.A. Logvinov, P.V. Gaman'kov, A.M. Timonov, Russ. J. Electrochem. 39 (2003) 310.

[20] I.A. Chepurnaya, P.V. Gaman'kov, T.Y. Rodyagina, S.V. Vasil'eva, A.M. Timonov, Russ. J. Electrochem. 39 (2003) 314.

[21] Y. Marcus, Chem. Rev. 88 (1988) 1475.

[22] R. Sakamoto, K.H. Wu, R. Matsuoka, H. Maeda, H. Nishihara, Chem. Soc. Rev. 44 (2015) 7698.

[23] M.F.S. Teixeira, T.R.L. Dadamos, Proc. Eurosensors Xxiii Conference 1 (2009) 297.

[24] V.V. Malev, O.V. Levin, A.M. Timonov, Electrochim. Acta 108 (2013) 313.

[25] M. Sharp, M. Petersson, K. Edstrom, J. Electroanal. Chem. 95 (1979) 123.

[26] H.C. Hurrell, H.D. Abruna, J. Electrochem. Soc. 135 (1988) C153.

[27] A.R. Guadalupe, D.A. Usifer, K.T. Potts, H.C. Hurrell, A.E. Mogstad, H.D. Abruna, J. Am. Chem. Soc. 110 (1988) 3462.

[28] H. Houjou, T. Motoyama, K. Araki, Eur. J. Inorg. Chem. (2009) 533.

[29] T.D.N. Minh, M.F. Charlot, A. Aukauloo, J. Phys. Chem. A 115 (2011) 911.

[30] H.C. Lin, N.W. Polaske, L.E. Oquendo, M. Gliboff, K.M. Knesting, D. Nordlund, D. S. Ginger, E.L. Ratcliff, B.M. Beam, N.R. Armstrong, D.V. McGrath, S.S. Saavedra, J. Phys. Chem. Lett. 3 (2012) 1154.

[31] H. Houjou, M. Ito, K. Araki, Inorg. Chem. 50 (2011) 5298.

[32] J. Tedim, S. Patricio, J. Fonseca, A.L. Magalhaes, C. Moura, A.R. Hillman, C. Freire, Synth. Met. 161 (2011) 680.

[33] O.S. Kutsenko, O.M. Burin, S.M. Maloletov, L.V. Tsymbal, Y.D. Lampeka, Teoreticheskaya I Eksperimentalnaya Khimiya 33 (1997) 165.

[34] C.S. Martin, M.F.S. Teixeira, Dalton Trans. 40 (2011) 7133.

[35] D.E. Schlamadinger, M.M. Daschbach, G.W. Gokel, J.E. Kim, J. Raman Spectrosc. 42 (2011) 633.

[36] K.S. Joya, X. Sala, Phys. Chem. Chem. Phys. 17 (2015) 21094.

[37] T.P. Gerasimova, S.A. Katsyuba, J. Organomet. Chem. 776 (2015) 30.

[38] E. Samuel, M. Bigorgne, J. Organomet. Chem. 30 (1971) 235.

[39] P. Sassi, M.L. Ceccarani, R.S. Cataliotti, Ber. Bunsen Ges. Phys. Chem. 99 (1995) 770.

[40] F.L. Lu, L. Rintoul, X. Sun, D.P. Arnold, X.X. Zhang, J.Z. Jiang, J. Raman Spectrosc. 35 (2004) 860. 